\title{
Recent developments: self-healing polymers based on quadruple hydrogen bonds
}

\author{
Guangpu Zhang ${ }^{1, \mathrm{a}}$, Zhe Sun ${ }^{1}$, Miaomiao $\mathrm{Li}^{2}$ \\ ${ }^{1}$ School of Chemistry and Chemical Engineering, Nanjing University of Science and Technology, Nanjing 210094, P. R. China \\ ${ }^{2}$ Shanghai Space Propulsion Technology Research Institute, Huzhou 313000, P. R. China
}

\begin{abstract}
The microcrack of materials was inevitable in the process of transportation, storage and utilization, which may cause functional failure and resources waste. Inspired by nature, self-healing polymers have attracted significant attention owing to widespread applications in wearable electronics, cartilage replacement, coatings and elastomer. Compared with extrinsic healing, intrinsically healable polymers offer multiple selfhealing by supramolecular reversible interactions, such as host-guest interactions, $\pi-\pi$ stacking, ionic interactions and hydrogen-bonding. Self-healing polymers based on quadruple hydrogen bonds have been extensively investigated due to its high thermodynamic stability and rapid kinetic reversibility, and have been well developed for the past two decades. In this paper, the strategies and designs of self-repairing polymers based on quadruple hydrogen bond were classified and summarized, including main-chain self-healing polymers, side-chain self-healing polymers and supramolecular self-healing polymers. It is expected that quadruple hydrogen bonding can be construct more robust, highly tough, multi-stimuli-responsive, and fast self-healing supramolecular polymer, and is potential to be applied to numerous civilian and military fields in the future.
\end{abstract}

\section{Introduction}

Microcrack and damage are inevitable in the process of material storage and utilization, which cause functional failure, resources waste, and even security issues ${ }^{[1,2]}$. Therefore, the ability to spontaneously heal defect is essential to engineering materials, and can improve their survivability, lifetime, and economic effect. Inspired by nature, like cucumbers ${ }^{[3]}$, mussel ${ }^{[4,5]}$, and titin $^{[6]}$, scientists and engineers have developed numerous self-healing materials over the past decades, which can be divided into extrinsic healing and intrinsic healing materials ${ }^{[7]}$. The latter, which includes host-guest interactions, $\pi-\pi$ stacking, ionic interactions and hydrogen-bonding, attracted public and scientific attention owing to its multiple self-healing and mild repairing condition.

Self-healing polymers based on hydrogen-bonding has been extensively investigated because of high affinity and directionality ${ }^{[8]}$. Ureidopyrimidone (UPy)(Figure1), which is a classic moiety of multiple $\mathrm{H}$-bonding, famous for its high thermodynamic stability $\left(\Delta G \approx 10 \mathrm{kcal} \mathrm{mol}^{-1}\right)$, rapid kinetic reversibility $\left(k_{\mathrm{off}} \approx 8 \quad \mathrm{~s}^{-1}\right)$ and high dimerization $\left(K_{\mathrm{dim}}=6 \times 10^{7} \mathrm{M}^{-1}\right.$, chloroform $)$, have been well developed these years ${ }^{[9-11]}$. Ureidopyrimidone (UPy) moiety can not only effectively enhance mechanical strength, but also endow engineering materials fascinating self-healing capacity, dramatically influence the safety, service life, intelligent manufacturing of the materials $^{[12,13]}$.
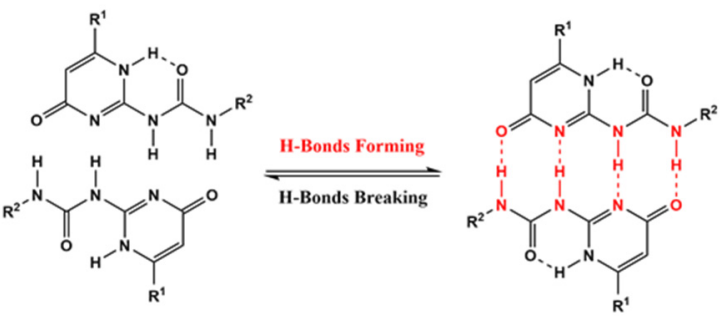

Figure 1. Self-repairing mechanism of Ureidopyrimidone (UPy) groups

Therefore, this paper summarized the research progress in self-healing polymers based on quadruple hydrogen bond, mainly including main-chain self-healing polymers, side-chain self-healing polymers and supramolecular self-healing polymers, and introduced the application prospect of self-healing polymers based on quadruple hydrogen bond. The future development of self-repairing materials involved smart material, composites material, and energetic materials is proposed. The mechanical properties, self-healing ability, and energy properties should be improved together with the introduction of quadruple hydrogen bond, indicating its potential for future technology development and industrial progress.

\section{Research progress of self-healing polymers based on quadruple hydrogen bonds}

The high dimerization and rapid kinetic reversibility of

\footnotetext{
${ }^{\mathrm{a}}$ Corresponding author: gpzhang@njust.edu.cn
} 
quadruple hydrogen bonds endow supramolecular polymers excellent mechanical strength and fascinating self-healing capability. The self-healing polymers based on quadruple hydrogen bonds can be spontaneously recovered original mechanical properties, electrical conductivity, and functional performance, improving material reliability, durability, and lifetime. The strategies and designs of self-healing polymers based on quadruple hydrogen bonds have been well pioneered and developed for the past two decades, which can be divided into main chain self-healing polymers, side chain self-healing polymers and supramolecular self-healing polymers according to the molecular structure and polymer designs.

\subsection{Main-chain self-healing polymer}

When main-chain self-healing polymer is damaged by external forces, the quadruple hydrogen bond between the polymer chains breaks first to form non-associative hydrogen bond groups. Under mild conditions, the quadruple hydrogen bond can be reformed to realize the self-healing of the material. Fan et al. introduced 2ureido- $4[1 \mathrm{H}]$-pyrimidinone (UPy) group into the PPG chain segment and prepared a robust self-healing polyurethane elastomer PPG-mUPy ${ }^{[14]}$. By adjusting the PPG segment length and UPy content, the tensile strength of optimized sample $\mathrm{PPG}_{1000}-\mathrm{mUPy}^{50 \%}$ can reach 20.62 $\mathrm{MPa}$, and the self-healing efficiency can reach over $93 \%$. The repair process is shown in the figure2. Yang et al. prepared waterborne polyurethane dispersion which used poly (1,4-buthylene-neopentylene adipate glycol) as soft segment, 2-ureido-4[1H]-pyrimidinone (UPy) functionalized monomer (5-(2-hydroxyethyl)-6-methyl2-aminouracil [HMA]), isophorone diisocyanate, blocking agent, and hydrophilic monomer as the hard segment ${ }^{[15]}$. The self-healing time of the scratched film is inversely proportional to the content of HMA, and the mechanical properties can be restored to more than $90 \%$ in $2.5 \mathrm{~h}$ at $80^{\circ} \mathrm{C}$. Wang et al. synthesized high-performance PUs with self-healing ability and reprocessability combining 2-ureido-4-[1H]-pyrimidinone (UPy) and iron-catechol (DOPA- $\mathrm{Fe}^{3+}$ ) cross-links ${ }^{[16]}$. These two dynamic forces, as sacrifice bonds, can effectively dissipate energy under external stress load, which makes the double-physical cross-linked network higher toughness and elongation at break. However, the selfhealing temperature of $\mathrm{PU}-\mathrm{UPy}-\mathrm{DOPA}-\mathrm{Fe}$ is $115^{\circ} \mathrm{C}$ and the self-repair efficiency is less than $80 \%$, which attributes to additional physical cross-linking structure formed by metal coordination. Fan et al. achieved visible light assisting supramolecular polymers combining dynamic ditelluride bonds and 2-ureido-4[1H]-pyrimidinone (UPy) moieties in main chain. Improved mechanical properties and fast recoverability attributes to the physical crosslinks formed by quadruple hydrogen bonded UPy moieties $^{[17]}$.
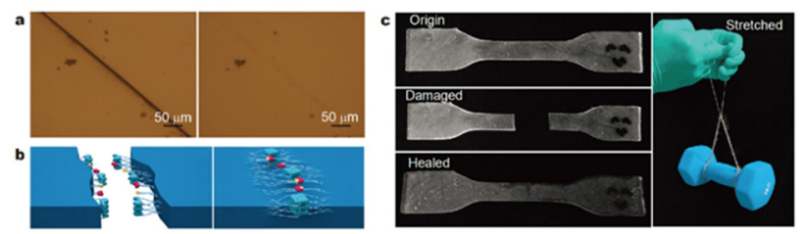

Figure 2. Self-healing process of PPG-mUPy

\subsection{Side-chain self-healing polymers}

Side-chain self-healing polymers can form a physical cross-linking network through the interaction of quadruple hydrogen bonds side groups, which can often be prepared by controllable free radical copolymerization or polyurethane condensation methods ${ }^{[18-21]}$. Zhu et al prepared self-healing polymers by free radical copolymerization of 2- (3- (6-methyl-4-oxo-1,4dihydropyrimidin-2-yl) ureido) ethyl acrylate (MAUPy), hydroxyethyl acrylate (HEA) , PEG360MA and PEG500MA ${ }^{[22]}$. The chain length of flexible chain determines the self-healing ability of the supramolecular polymer (Figure3). Specifically, a long flexible chain permits a lower glass transition temperature and sufficient chain segment, and thus ensure the self-healing properties at room temperature. Yan et al. developed a tough and multi-stimuli-responsive supramolecular polymer networks based on RAFT polymerization, which integrated the functions of the shape memory and selfhealing ${ }^{[23]}$. The polymer network consisted of two crosslinked networks, one is a chemical cross-linked network formed by the polymerization of PEG and PCL, and another is dynamic physical cross-linking network formed by the quadruple hydrogen bond of MAUPy. Chemical cross-linking and dynamic double cross-linking network, not only make it have dynamic reversible and selfrepairing properties, but also avoid permanent irreversible deformation under stress.

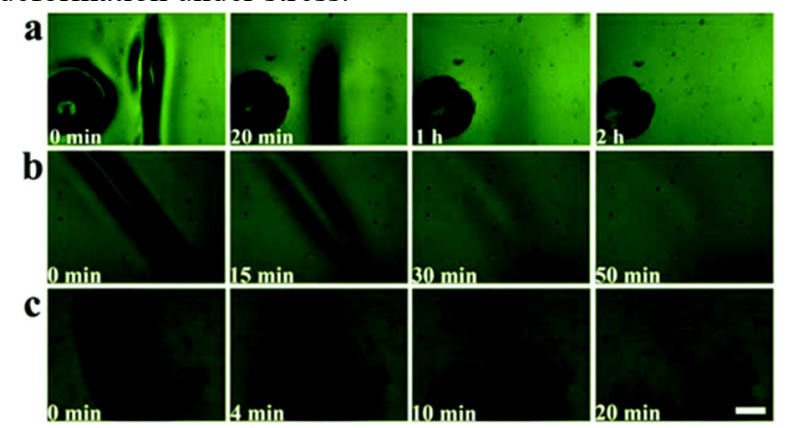

Figure 3. Self-healing processes of PHEA-UPy at ambient temperature with a relative humidity of $50 \%$ : a) PPEG360-

UPy; b) PPEG500-UPy films; and c) without special humidity treatment

Side-chain self-healing polymers can also be achieved by polycondensation of oligomer, isocyanate, and dihydroxyl chain extender with quadruple hydrogen bond ${ }^{[24-26]}$. Hu et al. reported a strategy of combining dynamic main chain and dangling UPy side group to fabricate a robust self-healing polyurethane ${ }^{[27]}$ (Figure4). When the content of quadruple hydrogen bonds is low, the effective binding of quadruple hydrogen bond will decrease and the self-repair property will decrease. If the content of quadruple hydrogen bond is high, the irregularity of structure and the blocking effect of 
hydrogen bond will be enhanced, and then the mechanical properties of quadruple hydrogen bond will be reduced. As the dangling side group, the quadruple hydrogen bond group intensified the chain diffusion ability at high temperature, and accelerated the recovery of tensile strength. Moreover, Side chain polymers can be obtained by the reactions of epoxide functionalized-PEG and 2(6isocyanatohexylaminocarbonylamino)-6-methyl-4[1H]pyrimidinone (UPy-NCO) with the hydroxyl of PVA reported by Ye Hyang Jo et al ${ }^{[25]}$. The optimal PVA-UPyPEG750 electrolyte material with low glass transition temperature $\left(T_{\mathrm{g}}\right)$, good self-healing ability and high ionic conductivity with shape memory. The PVA-UPy-PEG750 battery has good compatibility with lithium metal electrode and Li/PVA-UPy-PEG750/LFP can achieve coulomb efficiency of $99 \%$ with $0.1 \mathrm{C}$ at $60{ }^{\circ} \mathrm{C}$ and a discharge capacity of $145 \mathrm{~mA} \mathrm{~h} \mathrm{~g}^{-1}$.

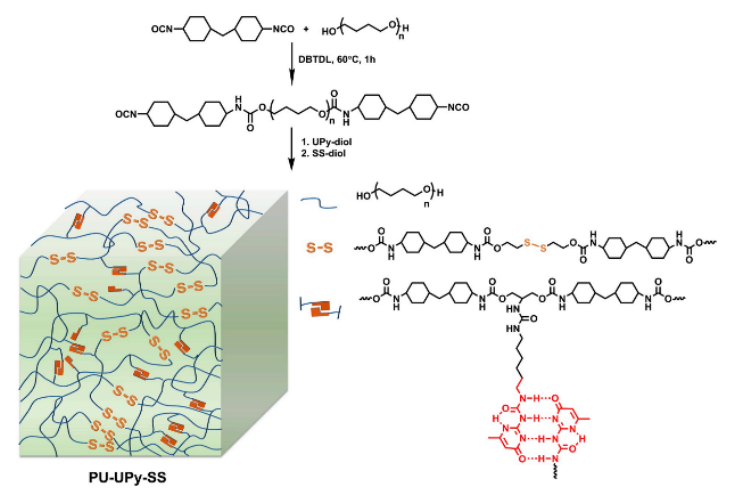

Figure 4. The synthetic routes of PU-UPy-SS samples with dynamic dangling UPy side chains

\subsection{Supramolecular Self-healing polymers}

The chain propagation of supramolecular self-healing polymer was achieved by quadruple hydrogen bond moiety, and the self-healing capability were accomplished by reversible UPy groups in the ends of the oligomer at both ends of the oligomer ${ }^{[28-36]}$. Yang et al. synthesized Ureidopyrimidinone functionalized polyethylene glycol (UPy-PEG-UPy) by mimicking the self-healing ability of living organisms and verified its application in anode materials for Li-ion batteries ${ }^{[37]}$. The self-healing electrode can be automatically repaired at room temperature after $1.5 \mathrm{~h}$ and exhibited excellent electrochemical performance, with an initial Coulomb efficiency of $81 \%$ and average capacity decay of only $0.04 \%$ after 400 cycles. Ren et al. prepared a linear polyurethane from UPy-Capped Poly(dl-Lactic Acid) Macrodiol, which exhibited attractive properties than covalent polyurethane ${ }^{[35]}$. The UPy-Capped polyurethane can be basically healed within $80 \mathrm{~s}$ and completely healed within $270 \mathrm{~s}$ at body temperature, and is expected to be applicated in the field of medical materials (Figure5). Dai et al. introduced the reversible imine bonds and quadruple hydrogen bonding (UPy) moieties into polymer networks to construct self-healable triboelectric nanogenerators based on infrared radiation through human skin ${ }^{[32]}$. Electrification layer almost completely recovered to the initial performance after $24 \mathrm{~h}$, which both imine bond and quadruple hydrogen bond contributes to remarkable selfhealing performance. KatelynR. Houston et al. investigated the effect of the UPy group, oligomer molecular weight on the structure-property relationships of supramolecular self-healing polymers by end-group functionalization of glycol modified PET with Ureidopyrimidinone [31]. The enhancement of materials properties of lower molecular weight polymer were remarkable, and the quadruple hydrogen bond with alkyl substituent can significantly improves the thermomechanical and viscoelastic properties due to the large substituent hindrance and competitive effect of aromatic ring substituent.

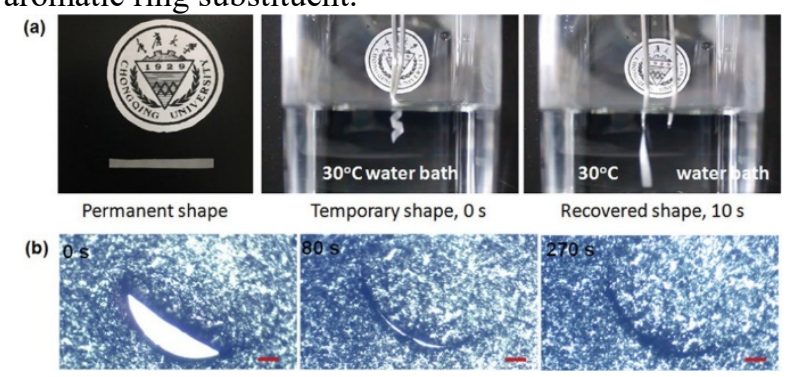

Figure 5. Shape memory effect and self-healing properties of UPy-PDLLA-PEG400-PDLLA4K-UPy

Bo Zhu et al. synthesized thermo-reversible supramolecular polyurethanes by condensation polymerization of isocyanate-terminated oligomer PTMG and 2-ureido-4-[1H]-pyrimidinone (UPy) with amine group $^{[38]}$. The UPy groups consequently serve as thermoreversible associations or the hard blocks and endow supramolecular polyurethane self-assemble capability and excellent mechanical properties. Bo Qin et al. proposed a new strategy of incorporating low-content quadruple hydrogen bonding to construct tough and multi-recyclable cross-linked supramolecular polyureas $^{[39]}$. Through the incorporation of quadruple hydrogen bonded diamine monomer, the transparent sample are endowed with superior toughness and excellent multiple recyclability which can recover more than $95 \%$ properties even after five generations of recycling processes (Figure6), providing a novel and general strategy for the preparation of reprocessible and recyclable cross-linked materials.

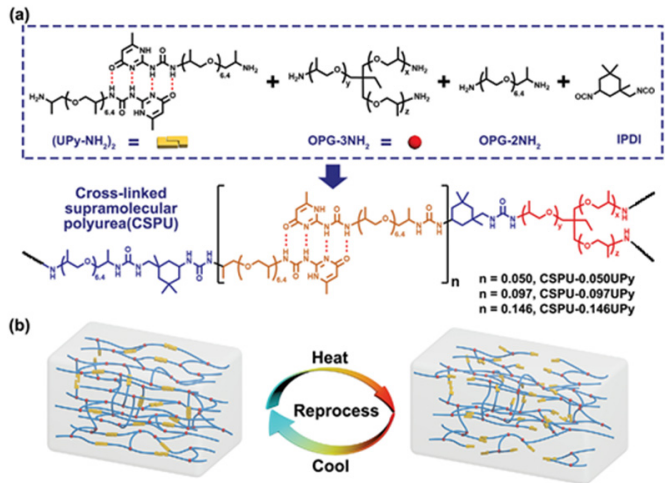

Figure 6. The synthesis, chemical structures, and schematic diagram of the recycling process of CSPU incorporating UPy groups

The above synthesis method required the precursor polymer with hydroxyl, or amine, or carboxylic groups, which also limits its application due to the difficult synthesize of UPy synthon. Sachin Bobade et al. 
combined ATRP and CuAAC click chemistry for the synthesis of telechelic oligomers functionalized with Propargyl-UPy and Azido-UPy ${ }^{[40]}$. The quadruple hydrogen bond provides the self-healing ability, and the triazole group can strengthen the UPy dimerization to enhance the mechanical properties of the polymer. However, complex copper used in $\mathrm{CuAAC}$ reaction were necessary to be removed by electrochemical avenue from the polymer $(>98 \%)$, for which standard polymer purification procedures fail ${ }^{[41]}$. $\mathrm{Xu}$ et al. realized the fabrication of cross-linked supramolecular polymers through photoinitiated thiol-ene click reaction of quadruple hydrogen bonded supramonomers ${ }^{[42]}$. It was found that the cross-linked supramolecular polymer has a higher critical polymerization concentration than the linear supramolecular polymer and the supramolecular interfacial polymerization can effectively control thermodynamic properties of the prepared quadruple hydrogen bond self-healing polymer ${ }^{[43]}$.

\section{Application}

The self-healing polymer based on quadruple hydrogen bonds, through the reformation of hydrogen bonds, endows the material self-repairing capacities which can be repaired at room temperature. It has significant influence in practical application, including self-healing hydrogels, self-healing elastomers, shape memory polymers based on $4 \mathrm{D}$ printing with longer service life, lower maintenance cost, and higher safety.

Hydrogel, an outstanding hydrophilic polymer, has been widely used in biomass materials, electronic sensors, and artificial organs, which attracted scientists in selfhealing field. Lin et al. successfully prepared a polyurethane hydrogel with UPy side groups by free radical copolymerization reaction ${ }^{[44]}$. The crack in hydrogel can be automatically repaired within 10 minutes at room temperature, and exhibited enhanced mechanical properties than traditional hydrogels (such as SR, NC, DN, and $\mathrm{MMC}$ hydrogels). Aránzazu del Campo et al. synthesized a self-healing based on quadruple hydrogen bonds with cracks almost can be disappeared within 2 minutes, representing an alternative to physical thermogelling hydrogels for injectable materials for biomedical applications $^{[20]}$. The sufficient chain movement provides hydrogels excellent self-healing, and the emergence of self-healing hydrogels show enormous potential in drug carriers, agricultural materials, food field.

Elastomer consists of soft matrix and hard phase, widely used in industrial products including rubber shoes, adhesives and electronic components. The emergence of self-healing technology endows elastomer fascinating character. Jian et al. synthesize a self-healing polyurethane elastomer which used PTMEG as the soft segment, IPDI as the hard segment, and HEDS as the chain extender. Disulfide exchange increase the mobility of the polymer chain, and hydrogen bond strengthen both mechanical properties and repairing capacity ${ }^{[45]}$. The repaired elastomer can reach to $5.01 \mathrm{MPa}$, almost recovering original tensile strength. Zhang et al. conclude self-healing capacity of polyurethane elastomer weaken when hard segment redundancy owing to insufficient hydrogen bonds. Song et al. prepared a self-healing elastomer based on quadruple hydrogen bonds in side chain. The toughness of the elastomer can reach 345 $\mathrm{MJ} / \mathrm{m}^{-3}$ after 48 hours of healing at $80^{\circ} \mathrm{C}$, showing its excellent self-healing effectiveness ${ }^{[46]}$. The application of self-healing elastomer undoubtedly exerts profound and lasting influence in future.

4D printing technology provides material capacity with the shape and structure can be dynamically changed over time, attracting intense attention all over the world ${ }^{[47]}$. However, the weak connection between the interfaces and terrible mechanical properties of printed products results in unsatisfactory printing structure and even printing failure. The introduction of self-healing technology into 4D printing can effectively resolve these defects ${ }^{[48,49]}$. Marta Invernizzi et al. explored digital light processing (DLP) printing with self-healing polymers based on quadruple hydrogen bonds ${ }^{[50,51]}$. The printed sample was shown in Figure7. The mechanical properties of printed samples are comparable to traditional processing products, and the self-healing efficiency is higher than that of cast samples. The self-healing of cracks can be achieved by placing it at $80^{\circ} \mathrm{C}$ for 1 hour, because the more effective interpenetration between PCLDMA and UPyMA results more quadruple hydrogen bonds in the damaged defect. Although the research on 4D printing based on quadruple hydrogen bonds is still in infancy, and the printing effect is not even ideal, however, the prospect is brightness due to the following advantages: first, it has greatly improved the mechanical properties, rheological properties, processability and other aspects of polymer materials; second, due to the dynamic reversibility of hydrogen bonds and the characteristics of macroscopic thermoplastics, polymers that could not be melt-processed (such as cross-linked resins) can now be processed by melt molding, which greatly expands the types of meltprocessed materials; Third, endowing the material with reproducible, self-repairing ability, and recycling characteristics.

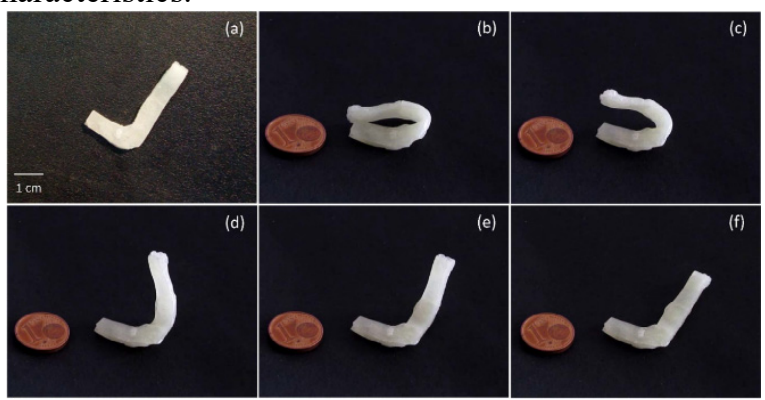

Figure 7. Shape memory effect of repaired samples by 4D printing

\section{Conclusion}

Self-healing polymers based on quadruple hydrogen bonds attracted public and scientific attention thanks to its excellent mechanical properties, charming self-healing capability, which can automatically repair damage by recombination of dynamic non-covalent quadruple hydrogen bonds under mild condition and improve the service life, stability and safety of engineering materials. 
Numerous endeavors have been dedicated to the research and exploration in self-healing polymers based on UPy groups, which can be classified into main chain, side chain, and supramolecular self-healing polymer according to the effect of UPy moieties in polymer chain. It was found that low prepolymer molecular weight, appropriate UPy content, and advisable polymer type could endow materials excellent self-healing efficiency. The combination of self-healing covalent bonds, crosslinking network structure could strengthen mechanical strength and broaden the application, however, these strategies may confine polymer chain movement and require higher temperature to achieve self-healing. The quadruple hydrogen bonding is expected to be construct more robust, highly tough, multi-stimuliresponsive, and fast self-healing supramolecular polymer by sophisticated molecular designing, and is potential to be applied to numerous civilian and military fields in the future.

\section{Acknowledgements}

This work was supported by the Natural Science Foundation of Jiangsu Province (BK20200471) and Industry-University-Research Cooperation Foundation of Shanghai Academy of Spaceflight Technology (SAST2020-093).

\section{References}

1. G. Chen, Y. Yu, X. Wu, G. Wang, J. Ren, Y. Zhao, Adv. Funct. Mater. 28, 1801386. (2018)

2. Z. Liu, Z. Zhang, R. O. Ritchie, Adv. Mater. 30, 1705220. (2018)

3. D. Yuan, S. Delpierre, K. Ke, J. Raquez, P. Dubois, I. Manas-Zloczower, ACS Appl. Mater. Inter. 11, 17853-17862. (2019)

4. S. Song, H. Yang, Y. Cui, Y. Tang, Y. Chen, B. Yang, J. Yuan, J. Huang, Polymer 198, 122528. (2020)

5. P. Zhao, C. Yin, Y. Zhang, X. Chen, B. Yang, J. Xia, L. Bian, J. Mater. Chem. A 8, 12463-12471. (2020)

6. J. Schuetz, W. Peng, P. Vana, Polym. Chem.-UK 6, 2150. (2015)

7. C. Heinzmann, C. Weder, L. M. de Espinosa, Chem. Soc. Rev. 45, 342-358. (2016)

8. Q. Pei, A. Ding, Prog. Chem. 31, 257-274 (2019)

9. G. Armstrong, M. Buggy, Materials Science \& Engineering C-Biomimetic and Supramolecular Systems 18, 45-49. (2001)

10. R. Sijbesma, F. Beijer, L. Brunsveld, B. Folmer, J. H. K. Hirschberg, R. Lange, J. Lowe, E. Meijer, Science (New York, N.Y.) 278, 1601-1604. (1997)

11. E. Berda, E. J. Foster, E. Meijer, Macromolecules 43. (2010)

12. S. C. Cummings, O. J. Dodo, A. C. Hull, B. Zhang, C. P. Myers, J. L. Sparks, D. Konkolewicz, ACS Applied Polymer Materials 2, 1108-1113. (2020)
13. L. Wang, Z. Gong, S. Li, W. Hong, Y. Zhong, D. Wang, L. Wan, Angewandte Chemie International Edition 55, 12393-12397. (2016)

14. C. Fan, Z. Huang, B. Li, W. Xiao, E. Zheng, K. Yang, Y. Wang, Science China Materials 62, 1188-1198. (2019)

15. Z. Yang, D. Sun, J. Appl. Polym. Sci. 137, 49413. (2020)

16. F. Wang, Z. Yang, J. Li, C. Zhang, P. Sun, ACS Macro Lett. 10, 510-517. (2021)

17. W. Fan, Y. Jin, L. Shi, W. Du, R. Zhou, S. Lai, Y. Shen, Y. Li, ACS Appl. Mater. Inter. 12, 6383-6395. (2020)

18. C. Heinzmann, U. Salz, N. Moszner, G. L. Fiore, C. Weder, ACS Appl. Mater. Inter. 7, 13395-13404. (2015)

19. Y. C. Gang Wanga, Angew. Chem. Int. Edit. 59, 2055-2060. (2019)

20. J. Cui, A. D. Campo, Chem. Commun. 48, 9302. (2012)

21. I. Jeon, J. Cui, W. R. K. Illeperuma, J. Aizenberg, J. J. Vlassak, Adv. Mater. 28, 4678-4683. (2016)

22. Q. Y. Dandan Zhu, Polym. Chem. 5086-5092. (2015)

23. M. L. Z. W. Jing Yan, Chem. Eng. J. 389, 123468. (2019)

24. Y. S. GuoLiang Li, Angew. Chem. Int. Edit. 57, 13838-13842. (2018)

25. B. Z. Ye Hyang Jo, Polym. Chem. 6561-6569. (2019)

26. Y. Chen, Y. Meng, Y. Luo, Y. Wang, Macromol. Mater. Eng. 305, 2000042. (2020)

27. J. Hu, R. Mo, X. Jiang, X. Sheng, X. Zhang, Polymer 183, 121912. (2019)

28. B. J. B. Folmer, R. P. Sijbesma, R. M. Versteegen, J. A. J. V. Rijt, E. W. Meijer, Adv. Mater. 12, 874-878. (2000)

29. R. Liu, X. Yang, Y. Yuan, J. Liu, X. Liu, Prog. Org. Coat. 101, 122-129. (2016)

30. B. Qin, S. Zhang, Z. Huang, J. F. Xu, X. Zhang, Macromolecules 8b-289b. (2018)

31. A. A. S. J. Katelyn R. Houston, Polym. Chem. 67446751. (2016)

32. X. Dai, L. B. Huang, Y. Du, J. Han, Q. Zheng, J. Kong, J. Hao, Adv. Funct. Mater. 1910723. (2020)

33. H. M. Keizer, R. V. Kessel, R. P. Sijbesma, E. W. Meijer, Polymer 44, 5505-5511. (2003)

34. F. Gao, J. Cao, Q. Wang, R. Liu, S. Zhang, J. Liu, X. Liu, Prog. Org. Coat. 113, 160-167. (2017)

35. W. Ren, Z. Li, Y. Chen, H. Gao, W. Yang, Y. Wang, Y. Luo, Macromol. Mater. Eng. 304, 1800491. (2019)

36. A. Watts, M. A. Hillmyer, Biomacromolecules 20, 2598-2609. (2019)

37. J. Yang, L. Zhang, T. Zhang, X. Wang, Y. Gao, Q. Fang, Electrochem. Commun. 87, 22-26. (2018)

38. B. Zhu, Z. Feng, Z. Zheng, X. Wang, J. Appl. Polym. Sci. 123, 1755-1763. (2012) 
39. B. Qin, S. Zhang, P. Sun, B. Tang, Z. Yin, X. Cao, Q. Chen, J. Xu, X. Zhang, Adv. Mater. 2000096. (2020)

40. S. Bobade, Y. Wang, J. Mays, D. Baskaran, Macromolecules 47, 5040-5050. (2014)

41. N. Jasinski, A. Lauer, P. J. M. Stals, S. Behrens, S. Essig, A. Walther, A. S. Goldmann, C. BarnerKowollik, ACS Macro Lett. 4, 298-301. (2015)

42. B. Q. J. X. Xiaoguang Liu, X. Z. Wang, Journal of Photochemistry and Photobiology A: Chemistry 355, 414-418. (2018)

43. S. Z. Bo Qin, Angew. Chem. Int. Edit. 56, 7639-7643. (2017)

44. Y. Lin, G. Li, J. Mater. Chem. B 2014, 2, 6878-6885.

45. X. Jian, Y. Hu, W. Zhou, L. Xiao, Polym. Advan. Technol. 29, 463-469. (2018)

46. Y. Song, Y. Liu, T. Qi, G. L. Li, Angewandte Chemie International Edition 57, 13838-13842. (2018)

47. H. WEI, X. WAN, Y. LIU, J. LENG, SCIENTIA SINICA Technologica 48, 2-16. (2018)

48. D. P. Street, W. K. Ledford, A. A. Allison, S. Patterson, D. L. Pickel, B. S. Lokitz, J. M. Messman, S. M. Kilbey, Macromolecules 52, 5574-5582. (2019)

49. Y. Zhu, J. Hu, Y. Liu, The European Physical Journal E 28, 3-10. (2009)

50. M. Invernizzi, S. Turri, M. Levi, R. Suriano, Eur. Polym. J. 101, 169-176. (2018)

51. R. Suriano, R. Bernasconi, L. Ma gagnin, M. Levi, J. Electrochem. Soc. 166, B3274- B3281. (2019) 